\title{
Real-space grid implementation of the projector augmented wave method
}

\author{
Mortensen, Jens Jørgen; Hansen, Lars Bruno; Jacobsen, Karsten Wedel
}

Published in:

Physical Review B Condensed Matter

Link to article, DOI:

10.1103/PhysRevB.71.035109

Publication date:

2005

Document Version

Publisher's PDF, also known as Version of record

Link back to DTU Orbit

Citation $(A P A)$ :

Mortensen, J. J., Hansen, L. B., \& Jacobsen, K. W. (2005). Real-space grid implementation of the projector augmented wave method. Physical Review B Condensed Matter, 71(3), 035109.

https://doi.org/10.1103/PhysRevB.71.035109

\section{General rights}

Copyright and moral rights for the publications made accessible in the public portal are retained by the authors and/or other copyright owners and it is a condition of accessing publications that users recognise and abide by the legal requirements associated with these rights.

- Users may download and print one copy of any publication from the public portal for the purpose of private study or research.

- You may not further distribute the material or use it for any profit-making activity or commercial gain

- You may freely distribute the URL identifying the publication in the public portal 


\title{
Real-space grid implementation of the projector augmented wave method
}

\author{
J. J. Mortensen, ${ }^{1}$ L. B. Hansen, ${ }^{1}$ and K. W. Jacobsen ${ }^{1}$ \\ ${ }^{1}$ CAMP and Department of Physics, Technical University of Denmark, DK-2800 Lyngby, Denmark
}

(Received 5 July 2004; published 21 January 2005)

\begin{abstract}
A grid-based real-space implementation of the projector augmented wave (PAW) method of Blöchl [Phys. Rev. B 50, 17953 (1994)] for density functional theory (DFT) calculations is presented. The use of uniform three-dimensional (3D) real-space grids for representing wave functions, densities, and potentials allows for flexible boundary conditions, efficient multigrid algorithms for solving Poisson and Kohn-Sham equations, and efficient parallelization using simple real-space domain-decomposition. We use the PAW method to perform all-electron calculations in the frozen core approximation, with smooth valence wave functions that can be represented on relatively coarse grids. We demonstrate the accuracy of the method by calculating the atomization energies of 20 small molecules, and the bulk modulus and lattice constants of bulk aluminum. We show that the approach in terms of computational efficiency is comparable to standard plane-wave methods, but the memory requirements are higher.
\end{abstract}

DOI: 10.1103/PhysRevB.71.035109

PACS number(s): 71.15.-m, 31.15.Ew, 31.15.Fx

\section{INTRODUCTION}

Density functional theory ${ }^{1,2}$ (DFT) combined with the generalized gradient approximation (GGA) for the exchange and correlation functional has become a popular method for studying materials and molecules at the atomic scale. Recently, there has been an increasing interest in using uniform real-space grids and finite-difference methods for doing DFT calculations. ${ }^{3-16}$ Real-space grids give an unbiased description of the wave functions, and the quality of the description can easily be controlled by changing the grid-point density. Finite-difference operators are used because the wavefunction values are given on grid points in real space and not in terms of a basis set. By doing all operations in real space, parallelization can be done by simple domain decomposition. ${ }^{6,17,18}$ Furthermore, real-space methods can make use of multigrid acceleration schemes ${ }^{19}$ for solving the Kohn-Sham equations ${ }^{2}$ and the Poisson equation. A further advantage of real space methods is the possibility for imposing localization constraints on the wave functions, which is the basis for linearly scaling electronic structure methods ${ }^{9,17}$ (order- $N$ methods).

Today, one of the most used methods for performing DFT calculations is the pseudopotential method using periodic supercells and plane-wave expansions for the pseudo-wave functions. This method shares with the grid-based methods the properties of unbiased representation of the wave functions and simple control of the quality of a calculation (by changing the number of plane waves). However, there are three major difficulties with a plane-wave representation for the wave functions. (i) Working with spatially localized wave functions, which is important for order- $N$ methods, is difficult with the extended nature of plane waves. (ii) Not all operations involving the wave functions, densities, and potentials can be done directly in the plane-wave representation, and Fourier transformations to and from real space must be carried out. Transformations between real and reciprocal spaces are highly nonlocal operations and, therefore, difficult to parallelize. (iii) Due to the periodicity of plane waves, the natural boundary conditions for a plane-wave calculation is periodic boundary conditions. Although all three problems have been addressed, ${ }^{20-24}$ the suggested solutions are not as simple as for grid-based approaches where all three problems have simple solutions.

An advantage of a plane-wave representation for the wave functions is its compactness. The memory footprint of a wave function is typically 10 times larger in a real-space grid representation compared to a plane-wave representation of similar accuracy. For this reason it is important to use soft pseudo-wave-functions that can be accurately represented on coarse grids. To our knowledge, until now, all applications of grid-based electronic structure calculations have used normconserving pseudopotentials. One way to get smoother pseudo-wave-functions is to relax the norm conservation of the wave functions and use ultrasoft pseudopotentials ${ }^{25,26}$ or the projector augmented wave (PAW) method. ${ }^{27,28}$ We have decided to use the PAW method. The PAW method works with soft-valence wave functions and, similar to the ultrasoft pseudopotential method, the wave functions need not be normalized. Contrary to the ultrasoft pseudopotential method, the PAW method is an all-electron method within the frozen core approximation, giving access to the true wave functions and the full electron density. The PAW method has been implemented for plane waves by several groups. ${ }^{27-32}$

We see the combination of real-space grid-based methods and the PAW method as an important step toward enabling larger calculations at a level of accuracy that is essentially all-electron in nature. There is a clear trend in electronic structure theory toward larger and more complex systems as for example nanostructures, large (bio-)molecular complexes and extended defects in real materials-systems that all quickly challenge present-day high-accuracy DFT codes, which are typically limited to, at most, a few hundred atoms. The great potential of the method presented here lies in the parallelization of the real-space algorithms. This makes it possible to make use of massively parallel computers as has been demonstrated by several other groups. ${ }^{6,17,18}$ In this paper, we focus on how to do accurate DFT calculations efficiently by using a real-space PAW method. We demonstrate the accuracy of our grid-based PAW calculations by showing 
that we are able to reproduce results for atomization energies from all-electron DFT calculations. This very stringent test shows that the methodology that we have developed is useful for real applications.

The solution of the Poisson equation is straightforward using multigrid methods ${ }^{19}$ (no Fourier transformations required). Solving the Kohn-Sham equations using multigrid methods is a much more difficult task; keeping the different eigenstates separated and orthogonal to each other can be a problem and representing the Hamiltonian on the coarse grids can also be problematic. We have decided to use the techniques typically used in state-of-the-art pseudopotential plane-wave calculations ${ }^{33}$ as they have been developed and improved over the the last few decades. For iteratively solving the Kohn-Sham equations, we use Pulay mixing techniques for obtaining the self-consistent density, ${ }^{33,34}$ subspace diagonalizations, and the residual minimization method ${ }^{33,35}$ using preconditioning of the electronic gradients for iteratively improving the wave functions. The preconditioning operation is a single multigrid $V$ cycle using only the kinetic energy operator as an approximate Hamiltonian. ${ }^{6}$

In Sec. II we will briefly summarize the PAW method. Then, in Sec. III, we will go through the details of our gridbased formulation of the PAW formalism. In Sec. IV, we describe how we solve the Kohn-Sham equations, and the evaluation of atomic forces is discussed in Sec. V. Section VI describes generalizations of the method to periodic systems with use of Brillouin zone sampling. In Sec. VII we apply the methodology that we have developed to a number of example systems and discuss approximations necessary for realistic calculations. Finally, in Sec. VIII we discuss the computational performance of our implementation. In the Appendices we provide explicit formulas needed for calculating the constants and functions that describe the atomic species.

\section{THE PROJECTOR AUGMENTED WAVE METHOD}

The notation we use is close to the one used by Blöchl in his papers on the PAW method..$^{27,28}$ We have used Hartree atomic units $(\hbar=m=e=1)$ and we write the equations for the case of a spin-paired and finite system of electrons.

The PAW method is based on a transformation between smooth pseudo-wave-functions, $\widetilde{\psi}_{n}$, and the true all-electron Kohn-Sham wave functions, $\psi_{n}$ ( $n$ is the band index). The core states of the atoms $\phi_{i}^{a, \text { core }}$ are frozen. Here $a$ is an atom index and $i$ is a combination of principal, angular momentum and magnetic quantum numbers, respectively $(n, \ell$, and $m)$.

Given a smooth pseudo-wave-function, the corresponding all-electron wave function, which is orthogonal to the set of $\phi_{i}^{a, \text { core }}$ orbitals, can be obtained through a linear transformation

$$
\psi_{n}(\mathbf{r})=\hat{\mathcal{T}} \widetilde{\psi}_{n}(\mathbf{r}) .
$$

The transformation operator $\hat{\mathcal{T}}$ is given in terms of atomcentered all-electron wave functions $\phi_{i}^{a}(\mathbf{r})$, the corresponding smooth partial waves $\widetilde{\phi}_{i}^{a}(\mathbf{r})$, and projector functions $\widetilde{p}_{i}^{a}(\mathbf{r})$, as

$$
\hat{\mathcal{T}}=1+\sum_{a} \sum_{i}\left(\left|\phi_{i}^{a}\right\rangle-\left|\widetilde{\phi}_{i}^{a}\right\rangle\right)\left\langle\widetilde{p}_{i}^{a}\right| .
$$

The atom-centered all-electron wave functions are taken from a calculation of a single atom with spherical symmetry: $\phi_{i}^{a}(\mathbf{r})=\phi_{n \ell}^{a}(r) Y_{L}(\hat{\mathbf{r}})$, where the $Y_{L}$ 's are real-valued spherical harmonics ( $L$ is a combined index for $\ell$ and $m$ ).

A radial cutoff distance, $r_{c}^{a}$, defining the atomic augmentation sphere is chosen. This radius is similar to a cutoff radius for a pseudopotential. The larger the augmentation sphere, the smoother the pseudo-wave-functions, but overlap with neighboring augmentation spheres must be avoided.

For all all-electron valence states, smooth partial waves $\widetilde{\phi}_{i}^{a}(\mathbf{r})=\widetilde{\phi}_{n \ell}^{a}(r) Y_{L}(\hat{\mathbf{r}})$ are constructed. The partial waves must match the corresponding all-electron waves for $r>r_{c}^{a}$. In this way, the correction in parenthesis in Eq. (2) is zero outside the augmentation spheres and we will have $\hat{\mathcal{T}}=1$ in this region. Note that there are no norm-conservation requirements to meet when choosing the shape of $\widetilde{\phi}_{n \ell}^{a}(r)$ inside the augmentation sphere.

Smooth projector functions must also be defined-one for each partial wave, $\widetilde{p}_{i}^{a}(\mathbf{r})=\widetilde{p}_{n \ell}^{a}(r) Y_{L}(\hat{\mathbf{r}})$. They must be localized inside the augmentation spheres and satisfy $\left\langle\widetilde{p}_{i_{1}}^{a} \mid \widetilde{\phi}_{i_{2}}^{a}\right\rangle$ $=\delta_{i_{1} i_{2}}$, which for the radial part gives

$$
\int_{o}^{r_{c}^{a}} r^{2} d r \widetilde{p}_{n \ell}^{a}(r) \widetilde{\phi}_{n^{\prime} \ell}^{a}(r)=\delta_{n n^{\prime}} .
$$

With this construction we have $\hat{\mathcal{T}} \widetilde{\phi}_{i}^{a}(\mathbf{r})=\phi_{i}^{a}(\mathbf{r})$.

In principle, an infinite number of projectors and partial waves are required for the PAW method to be exact. For practical calculations, a high-accuracy data set will need only one or two projector functions for each angular momentum channel of importance. This is similar to an ultrasoft pseudopotential, where a comparable number of projectors is needed. One partial wave is usually taken as the bound valence state, and additional waves can be taken from "excited states"- solutions to the radial Kohn-Sham equation at different noneigenvalue energies. The construction of partial waves and projector functions is described in Appendix A.

\section{A. PAW densities}

From the atomic frozen-core electron density $n_{c}^{a}(r)$, a smooth-core electron density $\widetilde{n}_{c}^{a}(r)$ is constructed, which must be identical to $n_{c}^{a}(r)$ for $r>r_{c}^{a}$. There is no normconservation requirement to meet when choosing the shape of $\widetilde{n}_{c}^{a}(r)$ inside the augmentation sphere.

The pseudoelectron density has contributions from the wave functions and from the atom-centered smooth-core electron densities

$$
\widetilde{n}(\mathbf{r})=\sum_{n} f_{n}\left|\widetilde{\psi}_{n}(\mathbf{r})\right|^{2}+\sum_{a} \widetilde{n}_{c}^{a}\left(\left|\mathbf{r}-\mathbf{R}^{a}\right|\right),
$$

where the $f_{n}$ 's are occupation numbers and $\mathbf{R}^{a}$ is the position of atom $a$.

An atomic density matrix [see Eq. (22) in Ref. 28] is defined as 


$$
D_{i_{1} i_{2}}^{a}=\sum_{n}\left\langle\widetilde{p}_{i_{1}}^{a} \mid \widetilde{\psi}_{n}\right\rangle f_{n}\left\langle\widetilde{\psi}_{n} \mid \widetilde{p}_{i_{2}}^{a}\right\rangle
$$

where

$$
P_{n i}^{a}=\left\langle\widetilde{p}_{i}^{a} \mid \widetilde{\psi}_{n}\right\rangle=\int d \mathbf{r} \widetilde{p}_{i}^{a}\left(\mathbf{r}-\mathbf{R}^{a}\right) \widetilde{\psi}_{n}(\mathbf{r}) .
$$

The PAW formalism defines atom-centered all-electron and pseudoelectron densities as

$$
n^{a}(\mathbf{r})=\sum_{i_{1} i_{2}} D_{i_{1} i_{2}}^{a} \phi_{i_{1}}^{a}(\mathbf{r}) \phi_{i_{2}}^{a}(\mathbf{r})+n_{\mathrm{c}}^{a}(r)
$$

and

$$
\widetilde{n}^{a}(\mathbf{r})=\sum_{i_{1} i_{2}} D_{i_{1} i_{2}}^{a} \widetilde{\phi}_{i_{1}}^{a}(\mathbf{r}) \widetilde{\phi}_{i_{2}}^{a}(\mathbf{r})+\widetilde{n}_{\mathrm{c}}^{a}(r),
$$

respectively. By construction, $n^{a}\left(\mathbf{r}-\mathbf{R}^{a}\right)$ is identical to the all-electron density $n(\mathbf{r})$, for $\left|\mathbf{r}-\mathbf{R}^{a}\right|<r_{c}^{a}$ and $\widetilde{n}^{a}\left(\mathbf{r}-\mathbf{R}^{a}\right)$ $=\widetilde{n}(\mathbf{r})$ for $\left|\mathbf{r}-\mathbf{R}^{a}\right|<r_{c}^{a}$ (see Ref. 27 for details). Therefore, the true all-electron density can be obtained from the pseudoelectron density

$$
n(\mathbf{r})=\widetilde{n}(\mathbf{r})+\sum_{a}\left[n^{a}\left(\mathbf{r}-\mathbf{R}^{a}\right)-\widetilde{n}^{a}\left(\mathbf{r}-\mathbf{R}^{a}\right)\right] .
$$

Again, the correction is zero outside the augmentation spheres.

A neutral charge density, $\widetilde{\rho}(\mathbf{r})$, is obtained by adding compensation charges, $\widetilde{Z}^{a}(\mathbf{r})$, inside the augmentation spheres to the pseudoelectron density. These charges compensate for the lack of norm-conservation and for the nuclear charge

$$
\widetilde{\rho}(\mathbf{r})=\widetilde{n}(\mathbf{r})+\sum_{a} \widetilde{Z}^{a}\left(\mathbf{r}-\mathbf{R}^{a}\right) .
$$

Using localized functions $\widetilde{g}_{L}^{a}(\mathbf{r})=\widetilde{g}_{\ell}^{a}(r) Y_{L}(\hat{\mathbf{r}})$ normalized as

$$
\int d \mathbf{r} r^{\ell} \widetilde{g}_{L}^{a}(\mathbf{r}) Y_{L}(\hat{\mathbf{r}})=1,
$$

the compensation charges are constructed with electrostatic multipole moments $Q_{L}^{a}$

$$
\widetilde{Z}^{a}(\mathbf{r})=\sum_{L} Q_{L}^{a} \widetilde{g}_{L}^{a}(\mathbf{r}) .
$$

The values to be used for the electrostatic multipole moments $Q_{L}^{a}$ are found by requiring the pseudo-charge-density, $\widetilde{n}^{a}+\widetilde{Z}^{a}$, to have the same electrostatic multipole moments as the all-electron charge density, $n^{a}+Z^{a}$, where $Z^{a}(\mathbf{r})=$ $-\mathcal{Z}^{a} \delta(\mathbf{r})$ is the nuclear charge density $\left(\mathcal{Z}^{a}\right.$ is the atomic number). This requirement can be expressed as

$$
\int d \mathbf{r} r^{\ell}\left[\widetilde{n}^{a}(\mathbf{r})+\widetilde{Z}^{a}(\mathbf{r})-n^{a}(\mathbf{r})-Z^{a}(\mathbf{r})\right] Y_{L}(\hat{\mathbf{r}})=0 .
$$

Inserting Eqs. (7), (8), and (12), we get

$$
Q_{L}^{a}=\sum_{i_{1} i_{2}} \Delta_{L i_{1} i_{2}}^{a} D_{i_{1} i_{2}}^{a}+\Delta^{a} \delta_{\ell 0},
$$

where the constants $\Delta_{L i_{1} i_{2}}^{a}$ and $\Delta^{a}$ are given by

$$
\Delta_{L i_{1} i_{2}}^{a}=\int d \mathbf{r} Y_{L}(\hat{\mathbf{r}}) r^{\ell}\left[\phi_{i_{1}}^{a}(\mathbf{r}) \phi_{i_{2}}^{a}(\mathbf{r})-\widetilde{\phi}_{i_{1}}^{a}(\mathbf{r}) \widetilde{\phi}_{i_{2}}^{a}(\mathbf{r})\right]
$$

and

$$
\Delta^{a}=\int d \mathbf{r} Y_{00}(\hat{\mathbf{r}})\left[-\mathcal{Z}^{a} \delta(\mathbf{r})+n_{c}^{a}(r)-\widetilde{n}_{c}^{a}(r)\right]
$$

\section{B. PAW total energy}

The PAW total energy is a function of the pseudo-wavefunctions $\widetilde{\psi}_{n}(\mathbf{r})$ and the occupation numbers $f_{n}$. The energy can be divided into a "soft" contribution $\widetilde{E}$ and corrections for each atom (see Refs. 27 and 28):

$$
E=\widetilde{E}+\sum_{a}\left(E^{a}-\widetilde{E}^{a}\right) .
$$

The soft energy contribution is

$$
\begin{aligned}
\widetilde{E}= & \sum_{n} f_{n} \int d \mathbf{r} \widetilde{\psi}_{n}^{*}(\mathbf{r})\left(-\frac{1}{2} \nabla^{2}\right) \widetilde{\psi}_{n}(\mathbf{r})+\frac{1}{2} \int d \mathbf{r} \widetilde{v}^{\mathrm{H}}(\mathbf{r}) \widetilde{\rho}(\mathbf{r}) \\
& +E_{\mathrm{xc}}[\widetilde{n}(\mathbf{r})]+\int d \mathbf{r} \widetilde{n}(\mathbf{r}) \sum_{a} \bar{v}^{a}\left(\left|\mathbf{r}-\mathbf{R}^{a}\right|\right),
\end{aligned}
$$

where $\widetilde{v}^{\mathrm{H}}(\mathbf{r})$ is the pseudo-Hartree potential, satisfying the Poisson equation $\nabla^{2} \widetilde{v}^{\mathrm{H}}=-4 \pi \widetilde{\rho}$, and $E_{\mathrm{xc}}$ is an exchangecorrelation functional. Finally, $\bar{v}^{a}(r)$ is an arbitrary localized potential vanishing for $r>r_{c}^{a}$. The soft energy contribution $\widetilde{E}$ is to be evaluated on three-dimensional grids in real space.

The atomic corrections to the energy $\left(E^{a}-\widetilde{E}^{a}\right)$ are given by

$$
\begin{aligned}
E^{a}= & \sum_{i}^{\text {core }} \int d \mathbf{r} \phi_{i}^{a, \text { core }}(\mathbf{r})\left(-\frac{1}{2} \nabla^{2}\right) \phi_{i}^{a, \text { core }}(\mathbf{r}) \\
& +\sum_{i_{1} i_{2}} D_{i_{1} i_{2}}^{a} \int d \mathbf{r} \phi_{i_{1}}^{a}(\mathbf{r})\left(-\frac{1}{2} \nabla^{2}\right) \phi_{i_{2}}^{a}(\mathbf{r}) \\
& +\frac{1}{2} \int d \mathbf{r} \int d \mathbf{r}^{\prime} \frac{\left[n^{a}(\mathbf{r})+Z^{a}(\mathbf{r})\right]\left[n^{a}\left(\mathbf{r}^{\prime}\right)+Z^{a}\left(\mathbf{r}^{\prime}\right)\right]}{\left|\mathbf{r}-\mathbf{r}^{\prime}\right|} \\
& +E_{\mathrm{xc}}\left[n^{a}(\mathbf{r})\right]
\end{aligned}
$$

and

$$
\begin{aligned}
\widetilde{E}^{a}= & \sum_{i_{1} i_{2}} D_{i_{1} i_{2}}^{a} \int d \mathbf{r} \widetilde{\phi}_{i_{1}}^{a}(\mathbf{r})\left(-\frac{1}{2} \nabla^{2}\right) \widetilde{\phi}_{i_{2}}^{a}(\mathbf{r}) \\
& +\frac{1}{2} \int d \mathbf{r} \int d \mathbf{r}^{\prime} \frac{\left[\widetilde{n}^{a}(\mathbf{r})+\widetilde{Z}^{a}(\mathbf{r})\right]\left[\widetilde{n}^{a}\left(\mathbf{r}^{\prime}\right)+\widetilde{Z}^{a}\left(\mathbf{r}^{\prime}\right)\right]}{\left|\mathbf{r}-\mathbf{r}^{\prime}\right|} \\
& +E_{\mathrm{xc}}\left[\widetilde{n}^{a}(\mathbf{r})\right]+\int d \mathbf{r} \widetilde{n}^{a}(\mathbf{r}) \bar{v}^{a}(r) .
\end{aligned}
$$

The energy contributions for $E^{a}$ and $\widetilde{E}^{a}$ are evaluated on radial grids inside the augmentation spheres.

By using Eqs. (7), (8), (12), and (14) we can reduce the atomic correction, $E^{a}-\widetilde{E}^{a}$, to a function of $D_{i_{1} i_{2}}^{a}$ 


$$
\begin{aligned}
E^{a}-\widetilde{E}^{a}= & A^{a}+\sum_{i_{1} i_{2}} B_{i_{1} i_{2}}^{a} D_{i_{1} i_{2}}^{a}+\sum_{i_{1} i_{2} i_{3} i_{4}} D_{i_{1} i_{2}}^{a} C_{i_{1} i_{2} i_{3} i_{4}}^{a} D_{i_{3} i_{4}}^{a} \\
& +\Delta E_{x c}^{a}\left(\left\{D_{i_{1} i_{2}}^{a}\right\}\right) .
\end{aligned}
$$

The constants $A^{a}, B_{i_{1} i_{2}}^{a}$, and $C_{i_{1} i_{2} i_{3} i_{4}}^{a}$ are evaluated in Appendix C.

The last term is an exchange-correlation correction

$$
\Delta E_{x c}^{a}\left(\left\{D_{i_{1} i_{2}}^{a}\right\}\right)=E_{\mathrm{xc}}\left[n^{a}(\mathbf{r})\right]-E_{\mathrm{xc}}\left[\widetilde{n}^{a}(\mathbf{r})\right],
$$

which is a function of $D_{i_{1} i_{2}}^{a}$ through Eqs. (7) and (8). For local and semilocal exchange-correlation functionals, $\Delta E_{x c}^{a}\left(\left\{D_{i_{1} i_{2}}^{a}\right\}\right)$ is written as an integration inside the augmentation sphere. There are several possibilities for the evaluation of this term. We use radial integration along lines from the center to a number of points distributed evenly on the surface of the augmentation sphere. ${ }^{29,36} \mathrm{We}$ find that this approach is the simplest for GGA functionals. ${ }^{37}$ Alternatively, one can expand the atomic densities in spherical harmonics, as described in Ref. 27 or use grid-free approaches. ${ }^{38,39}$

\section{UNIFORM 3D GRIDS}

In this section we give the details of our real-space gridbased implementation of the PAW method. From now on, wave functions, electron densities, and potentials are represented on three-dimensional uniform grids in real space.

\section{A. Localized functions and the double-grid technique}

In a grid representation, integrals over space are turned into sums over grid points. In the PAW method we often need to calculate the integral of a localized function, centered on an atom, multiplied by a function extended over all of space. As an example, let us take a projector function, $\widetilde{p}_{i}^{a}(\mathbf{r}$ $-\mathbf{R}^{a}$ ), centered on atom $a$ at position $\mathbf{R}^{a}$, multiplied by an extended wave function, $\widetilde{\psi}_{n}(\mathbf{r})$.

In the following, we use the index $G$ to index the grid points used for the wave functions. Transforming the integral to a sum over grid points $G$, with $V_{c}$ being the volume-pergrid point, we get

$$
P_{n i}^{a}=V_{c} \sum_{G} \tilde{p}_{i G}^{a}, \tilde{\psi}_{n G},
$$

where $\widetilde{\psi}_{n G}=\widetilde{\psi}_{n}\left(\mathbf{r}_{G}\right)$ and $\mathbf{r}_{G}$ is the position of grid point $G$ (only the grid points in the localized region around atom $a$ needs to be summed over). For $\widetilde{p}_{i G}^{a}$ we could use $\widetilde{p}_{i}^{a}\left(\mathbf{r}_{G}\right.$ $-\mathbf{R}^{a}$ ). However, this is not accurate enough, unless we use a very fine grid, which would compromise efficiency. Instead we use the elegant double-grid technique of Ono and Hirose. ${ }^{40}$ Here, the extended function is interpolated to a finer grid with grid points $f: \widetilde{\psi}_{n f}=\Sigma_{G} I_{f G} \widetilde{\psi}_{n G}$. The interpolation operator $I_{f G}$ takes the wave function from a coarse grid to a fine grid. Typically, a cubic interpolation is used and the fine grid has five times more points in each direction as the coarse grid. ${ }^{14,40}$ The localized projector function is evaluated on a fine grid as $\widetilde{p}_{i f}^{a}=\widetilde{p}_{i}^{a}\left(\mathbf{r}_{f}-\mathbf{R}^{a}\right)$. With $v$ being the volume per fine grid point, we get a more accurate sum

$$
v \sum_{f} \widetilde{p}_{i f}^{a} \widetilde{\psi}_{n f}=v \sum_{f} \widetilde{p}_{i f}^{a} \sum_{G} I_{f G} \widetilde{\psi}_{n G}=V_{c} \sum_{G}\left[\left(v / V_{c}\right) \sum_{f} I_{f G} \widetilde{p}_{i f}^{a}\right] \widetilde{\psi}_{n G} .
$$

Comparing the rightmost expression with Eq. (23), we identify the term in square brackets as the more accurate expression for $\widetilde{p}_{i G}^{a}$,

$$
\widetilde{p}_{i G}^{a}=\frac{v}{V_{c}} \sum_{f} I_{f G} \widetilde{p}_{i}^{a}\left(\mathbf{r}_{f}-\mathbf{R}^{a}\right) .
$$

This is equivalent to a restriction operation taking the localized function from the fine temporary grid to the coarse grid (restriction is the opposite of interpolation). Note that in Eq. (23), the sum is over coarse grid points and no actual interpolation of the extended function needs to be done. The evaluation of the localized function on the temporary grid is a relatively inexpensive operation, with an operation count that, for each atom, is independent of the system size.

We use the double-grid technique to transfer localized functions, such as $\widetilde{p}_{i}^{a}(\mathbf{r}), \widetilde{n}_{c}^{a}(r)$, and $\bar{v}^{a}(r)$, to values on realspace grids $\left(\widetilde{p}_{i G}^{a}, \widetilde{n}_{c G}^{a}\right.$, and $\left.\bar{v}_{g}^{a}\right)$.

\section{B. Real-space-grid formulation of the PAW method}

The formulas for densities, potentials, and energies, given in Sec. II, must be translated to a discretized form for use with a discrete representation of wave functions, densities, and potentials. The pseudoelectron density [Eq. (4)] is calculated on a coarse grid as

$$
\widetilde{n}_{G}=\sum_{n} f_{n}\left|\widetilde{\psi}_{n G}\right|^{2}+\sum_{a} \widetilde{n}_{c G}^{a} .
$$

The smooth atomic core electron densities can be chosen very soft, so that they can be added to the coarse grid.

The pseudoelectron density on the coarse grid is interpolated to a finer grid (grid points indexed by $g$ )

$$
\widetilde{n}_{g}=\sum_{G} I_{g G} \widetilde{n}_{G}
$$

We use a cubic interpolation for $I_{g G}$, and the fine grid has twice as many grid points as the coarse grid in each direction. From the pseudoelectron density on the fine grid, one can obtain the neutral charge density as [see Eq. (10)],

$$
\widetilde{\rho}_{g}=\widetilde{n}_{g}+\sum_{a} \widetilde{Z}_{g}^{a} .
$$

Using our grid representation for wave functions, densities, and potentials, we get for $\widetilde{E}$

$$
\begin{aligned}
\widetilde{E}= & \sum_{n} f_{n} V_{c} \sum_{G} \widetilde{\psi}_{n G}^{*} \sum_{G^{\prime}}\left(-\frac{1}{2} L_{G G^{\prime}}\right) \widetilde{\psi}_{n G^{\prime}}+\frac{1}{2} V_{f} \sum_{g} \widetilde{v}_{g}^{\mathrm{H}} \widetilde{\rho}_{g} \\
& +E_{\mathrm{xc}}\left(\left\{\widetilde{n}_{g}\right\}, V_{f}\right)+V_{f} \sum_{g} \widetilde{n}_{g} \sum_{a} \bar{v}_{g}^{a},
\end{aligned}
$$

where $V_{c}$ and $V_{f}$ are the volumes per grid point for the coarse wave-function grids and the fine density and potential grids, 
respectively, and $L_{G G^{\prime}}$ is a central finite-difference representation of the Laplacian. The discretization that we use for the Laplacian, uses a total of 12 neighbor points, giving an error of the order of $h^{6}$, where $h=V_{c}^{1 / 3}$ is the grid spacing.

The pseudo-Hartree potential $\widetilde{v}_{g}^{\mathrm{H}}$ is found by solving the Poisson equation, $\nabla^{2} \widetilde{v}^{\mathrm{H}}=-4 \pi \widetilde{\rho}$, using a discretization for the Laplacian

$$
\sum_{g^{\prime}} C_{g g^{\prime}} \widetilde{v}_{g^{\prime}}^{\mathrm{H}}=-4 \pi \widetilde{\rho}_{g} .
$$

This equation is solved using the multigrid technique pioneered by Brandt. ${ }^{19}$ Solving the Poisson equation iteratively on the finest grid will quickly reduce the short wavelength errors, but errors with larger wavelengths compared to the grid spacing are reduced only slowly. The multigrid technique introduces a series of coarser grids where the long wavelength errors can be effectively reduced.

We use a Mehrstellen discretization, ${ }^{6}$ where $C_{g g^{\prime}}$ $=\left(\mathbf{B}^{-1} \mathbf{A}\right)_{g g^{\prime}}$ is expressed in terms of two short-range finitedifference operators $A_{g g^{\prime}}$ and $B_{g g^{\prime}}$

$$
\sum_{g^{\prime}} A_{g g^{\prime}} \widetilde{v}_{g^{\prime}}^{\mathrm{H}}=-4 \pi \sum_{g^{\prime}} B_{g g^{\prime}} \widetilde{\rho}_{g^{\prime}} .
$$

For the coarse grids used in the multigrid $V$ cycle, simple nearest-neighbor central finite-difference Laplacians are used.

\section{Soft compensation charges}

Adding the compensation charges $\widetilde{Z}^{a}$ to the pseudoelectron density [Eqs. (10) and (28)] will require a very fine density grid in order to get an accurate description of the charge. The problem is that the compensation charges must be localized inside the augmentation spheres: $\widetilde{g}_{L}^{a}(\mathbf{r})=0$ for $r$ $>r_{c}^{a}$. Blöchl has described a method for plane-wave basis sets $^{27}$ that allows the use of softer compensation charges extending outside the augmentation spheres. We use the same method for our grid-based approach. A cutoff radius $\hat{r}_{c}^{a}$ larger than $r_{c}^{a}$ is chosen. Soft compensation charges with the same electrostatic multipole moments as the localized compensation charges are constructed

$$
\hat{Z}^{a}(\mathbf{r})=\sum_{L} Q_{L}^{a} \hat{g}_{L}^{a}(\mathbf{r}),
$$

where $\hat{g}_{L}^{a}(\mathbf{r})$ is a soft function localized within $r<\hat{r}_{c}^{a}$. The soft function $\hat{g}_{L}^{a}(\mathbf{r})$ is normalized in the same way as $\widetilde{g}_{L}^{a}(\mathbf{r})$ [see Eq. (11)].

Equation (28) must now be replaced by

$$
\widetilde{\rho}_{g}=\widetilde{n}_{g}+\sum_{a} \hat{Z}_{g}^{a}=\widetilde{n}_{g}+\sum_{a} \sum_{L} Q_{L}^{a} \hat{g}_{L g}^{a},
$$

and a correction must be added to $\widetilde{E}$. The correction is (see Ref. 27)

$$
\int d \mathbf{r} \widetilde{n}(\mathbf{r}) \sum_{a} \hat{v}^{a}\left(\mathbf{r}-\mathbf{R}^{a}\right)+\sum_{a a^{\prime}} U^{a a^{\prime}},
$$

where $\hat{v}^{a}(\mathbf{r})=\Sigma_{L} Q_{L}^{a} \hat{v}_{L}^{a}(\mathbf{r})$ and

$$
\hat{v}_{L}^{a}(\mathbf{r})=\int d \mathbf{r}^{\prime} \frac{\widetilde{g}_{L}^{a}\left(\mathbf{r}^{\prime}\right)-\hat{g}_{L}^{a}(\mathbf{r})}{\left|\mathbf{r}-\mathbf{r}^{\prime}\right|} .
$$

The first term in Eq. (34) is evaluated on the grid as $V_{f} \Sigma_{g} \tilde{n}_{g} \Sigma_{a} \hat{v}_{g}^{a}$. The transformation of the localized potential, $\hat{v}^{a}(\mathbf{r})$, and the localized compensation charge $\hat{Z}^{a}(\mathbf{r})$ (both vanishing for $r>\hat{r}_{c}^{a}$ ) to values at grid points $\hat{v}_{g}^{a}$ and $\hat{Z}_{g}^{a}$ is done using the double-grid technique. ${ }^{40}$ The last term in Eq. (34) is a pair potential with range $\hat{r}_{c}^{a}+\hat{r}_{c}^{a^{\prime}}$

$$
\begin{aligned}
U^{a a^{\prime}}= & \frac{1}{2} \int d \mathbf{r} \int d \mathbf{r}^{\prime}\left[\frac{\widetilde{Z}^{a}\left(\mathbf{r}-\mathbf{R}^{a}\right) \widetilde{Z}^{a^{\prime}}\left(\mathbf{r}^{\prime}-\mathbf{R}^{a^{\prime}}\right)}{\left|\mathbf{r}-\mathbf{r}^{\prime}\right|}\right. \\
& \left.-\frac{\hat{Z}^{a}\left(\mathbf{r}-\mathbf{R}^{a}\right) \hat{Z}^{a^{\prime}}\left(\mathbf{r}^{\prime}-\mathbf{R}^{a^{\prime}}\right)}{\left|\mathbf{r}-\mathbf{r}^{\prime}\right|}\right] \\
= & \frac{1}{2} \sum_{L L^{\prime}} Q_{L}^{a} V_{L L^{\prime}}^{a a^{\prime}} Q_{L^{\prime}}^{a^{\prime}},
\end{aligned}
$$

where

$$
\begin{aligned}
V_{L L^{\prime}}^{a a^{\prime}}= & \int d \mathbf{r} \int d \mathbf{r}^{\prime}\left(\frac{\widetilde{g}_{L}^{a}\left(\mathbf{r}-\mathbf{R}^{a}\right) \widetilde{g}_{L^{\prime}}^{a^{\prime}}\left(\mathbf{r}^{\prime}-\mathbf{R}^{a^{\prime}}\right)}{\left|\mathbf{r}-\mathbf{r}^{\prime}\right|}\right. \\
& \left.-\frac{\hat{g}_{L}^{a}\left(\mathbf{r}-\mathbf{R}^{a}\right) \hat{g}_{L^{\prime}}^{a^{\prime}}\left(\mathbf{r}^{\prime}-\mathbf{R}^{a^{\prime}}\right)}{\left|\mathbf{r}-\mathbf{r}^{\prime}\right|}\right) .
\end{aligned}
$$

The pair potential terms $V_{L L^{\prime}}^{a a^{\prime}}$ are functions of the difference vectors $\mathbf{R}^{a}-\mathbf{R}^{a^{\prime}}$.

\section{Orthogonality}

The orthogonality constraint of the all-electron wave functions, $\left\langle\psi_{n} \mid \psi_{n^{\prime}}\right\rangle=\delta_{n n^{\prime}}$, can be expressed in terms of the pseudo-wave-functions ${ }^{27}$ as $\left\langle\widetilde{\psi}_{n}|\hat{O}| \widetilde{\psi}_{n^{\prime}}\right\rangle=\delta_{n n^{\prime}}$, where the PAW overlap operator $\hat{O}$ is nonlocal

$$
\hat{O}=1+\sum_{a} \sum_{i_{1} i_{2}}\left|\widetilde{p}_{i_{1}}^{a}\right\rangle O_{i_{1} i_{2}}^{a}\left\langle\widetilde{p}_{i_{2}}^{a}\right|,
$$

with

$$
O_{i_{1} i_{2}}^{a}=\int d \mathbf{r}\left[\phi_{i_{1}}^{a}(\mathbf{r}) \phi_{i_{2}}^{a}(\mathbf{r})-\widetilde{\phi}_{i_{1}}^{a}(\mathbf{r}) \widetilde{\phi}_{i_{2}}^{a}(\mathbf{r})\right]=\sqrt{4 \pi} \Delta_{00 i_{1} i_{2}}^{a} .
$$

The discretized overlap operator looks like

$$
O_{G G^{\prime}}=\delta_{G G^{\prime}}+V_{c} \sum_{a} \sum_{i_{1} i_{2}} \widetilde{p}_{i_{1} G}^{a} O_{i_{1} i_{2}}^{a} \widetilde{p}_{i_{2} G^{\prime}}^{a},
$$

and the orthogonality constraint of the pseudo-wavefunctions can be expressed as

$$
V_{c} \sum_{G G^{\prime}} \widetilde{\psi}_{n G}^{*} O_{G G^{\prime}} \tilde{\psi}_{n^{\prime} G^{\prime}}=\delta_{n n^{\prime}} .
$$

\section{FINDING THE GROUND STATE}

In order to find the electronic ground state it is necessary to calculate the derivatives of the total energy with respect to 
wave-function values. This "electronic gradient" can be expressed in terms of a Hamiltonian $H_{G G^{\prime}}$,

$$
\frac{1}{V_{c}} \frac{\partial E}{\partial \widetilde{\psi}_{n G}^{*}}=f_{n} \sum_{G^{\prime}} H_{G G^{\prime}} \tilde{\psi}_{n G^{\prime}}
$$

The Hamiltonian is given as a sum of the kinetic energy operator and the local and nonlocal parts of the effective potential

$$
H_{G G^{\prime}}=-\frac{1}{2} L_{G G^{\prime}}+\widetilde{v}_{G} \delta_{G G^{\prime}}+V_{c} \sum_{a} \sum_{i_{1} i_{2}} \widetilde{p}_{i_{1} G}^{a} H_{i_{1} i_{2}}^{a} \widetilde{p}_{i_{2} G^{\prime}}^{a} .
$$

Explicit formulas for the local effective potential $\widetilde{v}_{G}$ and the "atomic" Hamiltonian, $H_{i_{1} i_{2}}^{a}$, are given in Appendix D.

The set of orthonormalized ground-state wave functions that diagonalize the Hamiltonian matrix $H_{n n^{\prime}}$ $=V_{c} \Sigma_{G G^{\prime}} \widetilde{\psi}_{n G^{*}}^{*} H_{G G^{\prime}} \widetilde{\psi}_{n^{\prime} G^{\prime}}$, must satisfy the Kohn-Sham equations

$$
\sum_{G^{\prime}}\left(H_{G G^{\prime}}-\epsilon_{n} O_{G G^{\prime}}\right) \tilde{\psi}_{n G^{\prime}}=0
$$

\section{A. Residual minimization method and Pulay mixing}

In order to locate the self-consistent ground state, we use the residual minimization method of Wood and Zunger ${ }^{35}$ (see also Ref. 33). The residuals are calculated as

$$
R_{n G}=\sum_{G^{\prime}}\left(H_{G G^{\prime}}-\epsilon_{n} O_{G G^{\prime}}\right) \tilde{\psi}_{n G^{\prime}},
$$

where $\epsilon_{n}$ is the current estimate of the eigenvalue of the $n$th band. Using a preconditioning operator $\hat{P}$ (to be discussed later), we can improve the wave function by taking a step along the direction of the preconditioned residual: $\widetilde{\psi}_{n G}$ $+\lambda \hat{P} R_{n G}$ ( $\lambda$ is the step length). The optimal step length is found by minimizing the norm of the residual for the new guess

$$
\begin{aligned}
R_{n G}^{\prime} & =\sum_{G^{\prime}}\left(H_{G G^{\prime}}-\epsilon_{n} O_{G G^{\prime}}\right)\left(\tilde{\psi}_{n G^{\prime}}+\lambda \hat{P} R_{n G^{\prime}}\right) \\
& =R_{n G}+\lambda \sum_{G^{\prime}}\left(H_{G G^{\prime}}-\epsilon_{n} O_{G G^{\prime}}\right) \hat{P} R_{n G^{\prime}} .
\end{aligned}
$$

Finding the optimal value for $\lambda$ amounts to finding the minimum of a second-order polynomial in $\lambda$. Having found the optimal step length for the first step, we do the actual update of the wave function by taking an additional step using the same step length in the direction of the preconditioned residual $R_{n G}^{\prime}$

$$
\tilde{\psi}_{n G} \leftarrow \tilde{\psi}_{n G}+\lambda \hat{P} R_{n G}+\lambda \hat{P} R_{n G}^{\prime} .
$$

For updating one wave function, one must apply the Hamiltonian twice and two preconditioning operations are required.

If we were to take steps along the residual (and not along the preconditioned residual), we would need very many it- erations in order to converge. The problem is that the residual vector is not necessarily parallel to the error vector (which we do not know). The purpose of preconditioning is to produce a direction that more accurately represents the error vector. ${ }^{41}$

We would get the optimal preconditioned residual $\widetilde{R}_{n}$ $=\hat{P} R_{n}$ by solving $\left(\hat{H}-\epsilon_{n} \hat{O}\right) \widetilde{R}_{n}=R_{n}$. Instead of solving this equation exactly, we solve approximately the simpler equation $-\frac{1}{2} \nabla^{2} \widetilde{R}_{n}=R_{n}$. This is done using one multigrid $V$ cycle, where a nearest-neighbor discretization is used for the Laplacian on the coarse grids. ${ }^{6}$

When all wave functions have been updated, the wave functions are orthonormalized and the density is updated. From the new density, a new Hamiltonian is generated $\left(\widetilde{v}_{G}\right.$ and $H_{i_{1} i_{2}}^{a}$ ). Finally, a subspace diagonalization is performed, and the next iteration toward self-consistency can begin. For each iteration a new input density is estimated using Pulay mixing. ${ }^{34}$ Typically three old densities are used. The atomic density matrices $D_{i_{1} i_{2}}^{a}$ are mixed as well. We start the iterations from a good guess at the wave function: a linear combination of atomic orbitals.

\section{FORCES}

The atomic force acting on an atom is defined as

$$
\begin{aligned}
\mathbf{F}^{a} & =-\frac{d E}{d \mathbf{R}^{a}}=-\frac{\partial E}{\partial \mathbf{R}^{a}}-\sum_{n G}\left(\frac{\partial E}{\partial \tilde{\psi}_{n G}} \frac{d \tilde{\psi}_{n G}}{d \mathbf{R}^{a}}+\text { c.c. }\right) \\
& =-\frac{\partial E}{\partial \mathbf{R}^{a}}-V_{c} \sum_{n} f_{n} \epsilon_{n} \sum_{G G^{\prime}} O_{G G^{\prime}}\left(\tilde{\psi}_{n G^{\prime}}^{*} \frac{d \tilde{\psi}_{n G}}{d \mathbf{R}^{a}}+\text { c.c. }\right) .
\end{aligned}
$$

In the last line, we have used Eqs. (42) and (44). The variation of the wave function corresponding to a variation in the position can be found from Eq. (41)

$$
\frac{d}{d \mathbf{R}^{a}} \sum_{G G^{\prime}} \widetilde{\psi}_{n G}^{*} O_{G G^{\prime}} \widetilde{\psi}_{n^{\prime} G^{\prime}}=0 .
$$

Inserting into Eq. (48), we get

$$
\begin{aligned}
\mathbf{F}^{a}= & -\frac{\partial E}{\partial \mathbf{R}^{a}}+V_{c} \sum_{n} f_{n} \epsilon_{n} \sum_{G G^{\prime}} \tilde{\psi}_{n G}^{*} \frac{d O_{G G^{\prime}}}{d \mathbf{R}^{a}} \tilde{\psi}_{n G^{\prime}} \\
= & -V_{c} \sum_{G} \tilde{v}_{G} \frac{d \widetilde{n}_{c G}^{a}}{d \mathbf{R}^{a}}-V_{f} \sum_{g} \tilde{v}_{g}^{H} \sum_{L} Q_{L}^{a} \frac{d \hat{g}_{L g}^{a}}{d \mathbf{R}^{a}} \\
& -V_{f} \sum_{g} \tilde{n}_{g}\left(\frac{d \bar{v}_{g}^{a}}{d \mathbf{R}^{a}}+\sum_{L} Q_{L}^{a} \frac{d \hat{v}_{L g}^{a}}{d \mathbf{R}^{a}}\right) \\
& -V_{c} \sum_{n} f_{n} \sum_{i_{1} i_{2}}\left(H_{i_{1} i_{2}}^{a}-\epsilon_{n} O_{i_{1} i_{2}}^{a}\right) \\
& \times \sum_{G}\left[\tilde{\psi}_{n G} \frac{d \widetilde{p}_{i_{1} G}^{a}}{d \mathbf{R}^{a}}\left(P_{n i_{2}}^{a}\right)^{*}+\text { c.c. }\right]-\sum_{a^{\prime}} \sum_{L L^{\prime}} Q_{L}^{a} \frac{d V_{L L^{\prime}}^{a a^{\prime}}}{d \mathbf{R}^{a}} Q_{L^{\prime}}^{a^{\prime}}
\end{aligned}
$$




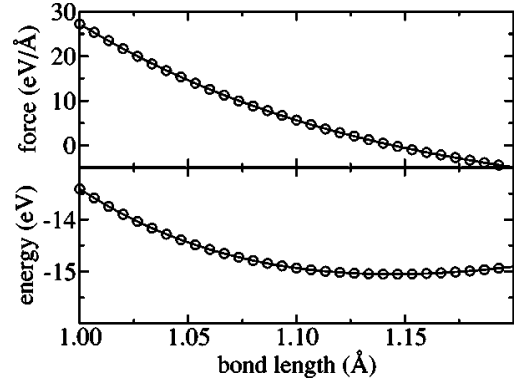

FIG. 1. Energy and force for a CO molecule at different bond lengths calculated with $h=0.2 \AA$. Bottom: The circles show the calculated energies and the curve shows a third-order polynomial fit. Top: The circles show the calculated forces according to Eq. (50). The curve is minus the derivative of the third-order polynomial fit to the energies.

As an example, we show in Fig. 1, the force along the bond of a $\mathrm{CO}$ molecule calculated using the analytical expression above. Fitting a third-order polynomial to the energies and taking the negative of the derivative with respect to the bond length is seen to give exactly the same force.

\section{GENERALIZATIONS}

We have implemented the algorithms introduced above, and in addition we have made two extensions: (i) treatment of spin-polarized systems and (ii) treatment of periodic systems using Brillouin-zone sampling. The first extension is straightforward. When $\mathbf{k}$ points are introduced in order to treat periodic systems, we can work directly with the wave functions and use Bloch boundary conditions ${ }^{42}$

$$
\widetilde{\psi}_{n \mathbf{k}}(\mathbf{r}+\mathbf{R})=e^{i \mathbf{k} \cdot \mathbf{R}} \tilde{\psi}_{n \mathbf{k}}(\mathbf{r}),
$$

where $\mathbf{R}$ is any Bravais lattice vector. This is different from the plane-wave approach, where the periodic basis set forces one to work with the periodic part of the wave function and the Hamiltonian becomes k-point dependent. In our case, the boundary conditions become k-point dependent.

It is only necessary to work with the $\mathbf{k}$ points in the irreducible part of the Brillouin zone. Each $\mathbf{k}$ point will have a specific weight, and densities, atomic density matrices, and forces should be appropriately symmetrized.

For evaluating the GGA exchange-correlation energy and potential, we use a finite-difference operator for calculating the gradient of the density. The exchange-correlation potential is calculated as the exact derivative of the discretised exchange-correlation energy with respect to $\tilde{n}_{g}$ (similar in spirit to the method of White and Bird ${ }^{43}$ used for plane-wave basis sets).

\section{APPLICATIONS}

The first application of the algorithms described here is the calculation of atomization energies for the 20 small molecules listed in Table I using the PBE (Ref. 44) exchangecorrelation functional. The augmentation sphere radii used and the number of projectors used are shown in Table II. The
TABLE I. PBE and experimental atomization energies [experimental geometries are used (Ref. 44), and zero point vibration energy has been removed]. The ground states of $\mathrm{C}, \mathrm{O}, \mathrm{F}, \mathrm{P}$, and $\mathrm{Cl}$ are found to be nonspherical (Ref. 45). All-electron and experimental numbers are taken from Refs. 46 and 47.

\begin{tabular}{|c|c|c|c|c|}
\hline \multirow[b]{2}{*}{ Molecule } & \multicolumn{3}{|c|}{ PBE } & \multirow[b]{2}{*}{ Experiment $^{\mathrm{a}}$} \\
\hline & PAW & all-electron $^{\mathrm{a}}$ & all-electron $^{\mathrm{b}}$ & \\
\hline $\mathrm{H}_{2}$ & 4.52 & 4.54 & 4.53 & 4.75 \\
\hline $\mathrm{LiH}$ & 2.32 & 2.32 & 2.32 & 2.51 \\
\hline $\mathrm{CH}_{4}$ & 18.17 & 18.20 & 18.18 & 18.18 \\
\hline $\mathrm{NH}_{3}$ & 13.03 & 13.08 & 13.05 & 12.90 \\
\hline $\mathrm{OH}$ & 4.76 & 4.76 & 4.75 & 4.61 \\
\hline $\mathrm{H}_{2} \mathrm{O}$ & 10.14 & 10.16 & 10.14 & 10.07 \\
\hline $\mathrm{HF}$ & 6.27 & 6.16 & 6.14 & 6.11 \\
\hline $\mathrm{Li}_{2}$ & 0.89 & 0.86 & 0.85 & 1.06 \\
\hline $\mathrm{LiF}$ & 6.16 & 6.01 & 6.05 & 6.02 \\
\hline $\mathrm{Be}_{2}$ & 0.35 & 0.42 & 0.41 & 0.13 \\
\hline $\mathrm{C}_{2} \mathrm{H}_{2}$ & 17.95 & 17.99 & 17.91 & 17.58 \\
\hline $\mathrm{C}_{2} \mathrm{H}_{4}$ & 24.75 & 24.78 & 24.73 & 24.40 \\
\hline $\mathrm{HCN}$ & 14.10 & 14.14 & 14.07 & 13.53 \\
\hline $\mathrm{CO}$ & 11.58 & 11.66 & 11.60 & 11.24 \\
\hline $\mathrm{N}_{2}$ & 10.41 & 10.55 & 10.46 & 9.91 \\
\hline NO & 7.38 & 7.45 & 7.36 & 6.63 \\
\hline $\mathrm{O}_{2}$ & 6.27 & 6.23 & 6.14 & 5.23 \\
\hline $\mathrm{F}_{2}$ & 2.28 & 2.32 & 2.25 & 1.67 \\
\hline $\mathrm{P}_{2}$ & 5.18 & 5.25 & 5.08 & 5.09 \\
\hline $\mathrm{Cl}_{2}$ & 2.83 & 2.82 & 2.74 & 2.52 \\
\hline
\end{tabular}

Reference 46.

${ }^{b}$ Reference 47.

choice of the augmentation sphere radii is a compromise between smooth pseudo-wave-functions and a low number of projector functions. A larger radius will allow us to have smoother pseudo-wave-functions, but more projector functions will be needed for high accuracy. Furthermore, the radius is limited by the requirement that the overlap between neighboring augmentation spheres should be small. The radii we have chosen will give slight overlaps in some of the molecular calculations.

For the second-row atoms, the $1 s$ orbital is treated as a core state and frozen, and for the third-row atoms, the $1 s, 2 s$, and $2 p$ orbitals are frozen. For hydrogen, lithium, and beryllium, we use two $s$ projectors and one $p$ projector, and for the rest of the atoms two $s$ projectors, two $p$ projectors, and one $d$ projector are used. The compensation charges were taken to be spherical. We calculate the atomic exchange-correlation correction energy, Eq. (22), using 49 line integrations in each sphere. ${ }^{36}$ All calculations are done using periodic boundary conditions.

With these approximations we get excellent agreement with full all-electron calculations (see Table I). The average and maximum differences between our PAW atomization energies and the all-electron calculations of Kurth et al. ${ }^{46}$ are $0.05 \mathrm{eV}$ and $0.15 \mathrm{eV}$, respectively, and comparing to the all- 
TABLE II. Augmentation sphere radii in atomic units and number of projectors.

\begin{tabular}{|c|c|c|c|c|c|c|c|c|c|c|c|}
\hline Atom & $\mathrm{H}$ & $\mathrm{Li}$ & $\mathrm{Be}$ & $\mathrm{C}$ & $\mathrm{N}$ & $\mathrm{O}$ & $\mathrm{F}$ & $\mathrm{Al}$ & $\mathrm{Si}$ & $P$ & $\mathrm{Cl}$ \\
\hline$r_{c}^{a}$ (Bohr) & 0.9 & 1.5 & 1.5 & 1.0 & 1.1 & 1.2 & 1.2 & 2.0 & 2.0 & 2.0 & 1.5 \\
\hline Number of projectors & $s^{2} p$ & $s^{2} p$ & $s^{2} p$ & $s^{2} p^{2} d$ & $s^{2} p^{2} d$ & $s^{2} p^{2} d$ & $s^{2} p^{2} d$ & $s^{2} p^{2} d$ & $s^{2} p^{2} d$ & $s^{2} p^{2} d$ & $s^{2} p^{2} d$ \\
\hline
\end{tabular}

electron calculations of Zhang et al.,${ }^{47}$ we get an average difference of $0.05 \mathrm{eV}$ and a maximum difference of $0.13 \mathrm{eV}$ (the two sets of all-electron calculations differ by $0.05 \mathrm{eV}$ in average and 0.17 as maximum). We find that all atomization energies are converged to within $0.03 \mathrm{eV} /$ atom at a grid spacing of $0.1875 \AA$.

Interestingly, we find the convergence of total energies with respect to grid spacing to be very systematic. Figure 2 shows the atomization energy of nitrogen as a function of the fourth power of the grid spacing. It is seen that for small $h$, all points fall exactly on a straight line, which allows us to extrapolate energies to the limit of an infinitely dense grid $(h=0)$. The PAW numbers presented in Table I have been extrapolated to $h=0$. A similarly transparent convergence of DFT calculations was recently observed by Daykov et al. ${ }^{48}$ for wavelet-based calculations. A quartic convergence is to be expected because all approximations are accurate to order at least $h^{3}$.

Figure 3 shows the variation of the energy as a hydrogen atom is displaced from one grid point to a neighboring grid point. Ideally, there should be no variation (we are using periodic boundary conditions). In practice, we have to make sure that this energy variation and the corresponding forces are acceptably small. For hydrogen, the variation is below $0.15 \mathrm{meV}$ (full line in Fig. 3). The energy varies periodically with period $h$. Note that there is also a modulation of the energy with a period of $h / 5$. This stems from the Ono-Hirose restriction of the projector functions; the localized projector functions are evaluated on a fine grid with a grid spacing of $h / 5$ and then restricted to a coarse grid with the same grid density as the wave functions. The oscillations give rise to forces up to $0.006 \mathrm{eV} / \AA$ in magnitude, which is acceptable for most applications. The forces can be reduced further either by using a finer grid for the wave functions or by using a finer grid for the Ono-Hirose restriction of the projector functions. If the projectors are evaluated directly on the coarse grid, then the variation of the energy is $55 \mathrm{meV}$-more than two orders of magnitude larger (dashed line in Fig. 3). This clearly demonstrates the importance of the Ono-Hirose restriction.

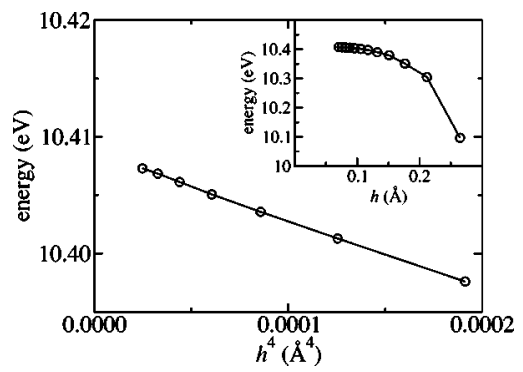

FIG. 2. Atomization energy of a nitrogen molecule as a function of $h^{4}$. The inset shows the atomization energy as a function of $h$.
For the bulk aluminum calculation [LDA (Ref. 49) and PBE (Ref. 44) results are shown in Table III], we use a cubic unit cell containing four aluminum atoms and use $10 \times 10$ $\times 10 \mathbf{k}$ points. Again, we get good agreement with exact all-electron calculations for both the lattice constant and bulk modulus (the bulk modulus is calculated at the theoretical lattice constant).

\section{PERFORMANCE}

We have compared the performance of the real-space code to a highly optimized ultrasoft plane-wave code. ${ }^{50-52}$ The ground state of $64 \mathrm{Si}$ atoms in the diamond structure was found using both codes. For the plane-wave calculation, plane waves with kinetic energies up to $100 \mathrm{eV}$ were used and the size of the real-space grid for fast Fourier transforms was $36 \times 36 \times 36$ points. The same grid size was used for the real-space code $(h=0.30 \AA)$. After finding the electronic ground state, one atom was displaced by $0.1 \AA$, and the time for converging to the new ground state was measured. The measured times were 21 and $26 \mathrm{~min}$ for the plane-wave and real-space codes, respectively, on a Pentium- $42.6 \mathrm{GHz}$ Linux machine. We have estimated the degree of convergence of the two codes by calculating the cohesive energy of silicon. The cohesive energy calculated with the plane-wave code and a plane-wave cutoff of $100 \mathrm{eV}$ was converged to within $40 \mathrm{meV}$ of the fully converged value. With the realspace code and $h=0.30 \AA$, the cohesive energy was converged to within $3 \mathrm{meV}$ of the $h=0$ value. This and similar results for other calculations that we have performed seem to indicate that the real-space code obtains a somewhat better convergence at the same grid spacing as the plane-wave code. Furthermore, the real-space code can, most likely, take advantage of a number of improvements, such as algorithmic improvements and optimizations of floating point operations and memory access. The plane-wave code has already benefited from optimizations of this sort.

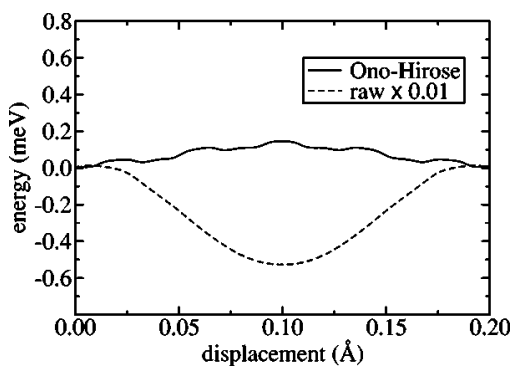

FIG. 3. Energy variation as a hydrogen atom is displaced from one grid point to a neighboring grid point for $h=0.2 \AA$. The full and dashed curves show the result with and without using the doublegrid technique (note that the dashed curve has been multiplied by $0.01)$. 
TABLE III. Lattice constant and bulk modulus for fcc bulk aluminum. All-electron and experimental numbers are taken from Ref. 46.

\begin{tabular}{ccccc}
\hline \hline & \multicolumn{2}{c}{$a(\AA)$} & \multicolumn{2}{c}{$B(\mathrm{GPa})$} \\
XC & PAW & all-electron & PAW & all-electron \\
\hline LDA & 3.987 & 3.983 & 83.6 & 84.0 \\
PBE & 4.043 & 4.039 & 77.7 & 77.3 \\
experiment & \multicolumn{2}{c}{4.050} & \multicolumn{2}{c}{77.3} \\
\hline
\end{tabular}

Regarding the memory requirements, the plane-wave code is clearly more economic. The memory required to store one wave function for the 64 atom silicon system is $36^{3}=46656$ floating point numbers for the real-space code and 2897 floating point numbers ${ }^{53}$ for the plane-wave code.

\section{DISCUSSION}

Using the techniques described in this paper, an important step in every electronic structure calculation, namely, the application of the Hamiltonian to all wave functions, can be done in $O\left(N^{2}\right)$ operations. This is a more optimal scaling than the $O\left(N^{2} \log N\right)$ scaling, which can be achieved with plane-wave basis sets and fast Fourier transforms. Operations such as orthogonalization and subspace diagonalization of the wave functions scale as $O\left(N^{3}\right)$. Luckily, the prefactors for these $O\left(N^{3}\right)$ operations are very low, so that very large system sizes are required before the $O\left(N^{3}\right)$ terms become the bottleneck. ${ }^{54}$ In the limit where $O\left(N^{3}\right)$ terms start to dominate, plane-wave methods will, in principle, have an advantage because the number of plane-wave coefficients will typically be less than the number of grid points used in realspace grid-based calculations. However, we believe that, for those very large systems, efficient parallelization on massively parallel computers and use of $O(N)$ methods are crucial.

Currently, we have a single-processor implementation of our PAW real-space algorithms. ${ }^{55}$ This limits us to study rather small systems. Obviously a parallelization using realspace domain decomposition is needed. For the small systems that we have studied thus far, charge sloshing is less of a problem, but it may become a problem when we move on to larger systems. It will therefore be necessary to improve on our mixing of the density. A preconditioning, that will damp the long wavelength changes to the density, such as that proposed by Kerker, ${ }^{56}$ should be considered. Another improvement would be to use a special metric, which weights long wavelength errors higher than short wavelength errors, for estimating the norm of the difference between input and output densities in the Pulay method. ${ }^{33}$ These improvements to the density mixing are easily implemented in reciprocal space, but may be challenging to do in real space.

\section{ACKNOWLEDGMENTS}

Financial support from the Carlsberg Foundation is gratefully acknowledged. We furthermore acknowledge support from the Materials Research Program of the Danish Research Agency (Grant No. 5020-00-0012) and the Danish Center for Scientific Computing.

\section{APPENDIX A: CONSTRUCTION OF PARTIAL WAVES AND PROJECTOR FUNCTIONS}

A DFT calculation for the atom is performed using radial grids for the wave functions, densities, and potentials. The radial Kohn-Sham equation gives us a set of radial allelectron wave functions, normalized as $\int r^{2} d r\left[\phi_{n \ell}^{a}(r)\right]^{2}=1$. The core states are only used for constructing a frozen-core electron density

$$
n_{c}^{a}(r)=2 \sum_{n \ell}^{\text {core }} \frac{2 \ell+1}{4 \pi}\left[\phi_{n \ell}^{a, \text { core }}(r)\right]^{2} .
$$

The smooth partial wave functions are chosen as

$$
\widetilde{\phi}_{n \ell}^{a}(r)=\sum_{i=0}^{3} c_{i} r^{2 i}
$$

for $r<r_{c}^{a}$, and the coefficients $c_{i}$ are chosen so that $\widetilde{\phi}_{n \ell}^{a}$ joins $\phi_{n \ell}^{a}$ smoothly at $r=r_{c}^{a}$. The projector functions are calculated as

$$
\tilde{p}_{n \ell}^{a}(r)=\left(-\frac{1}{2} \nabla^{2}+\widetilde{v}(r)-\epsilon_{n \ell}^{a}\right) \widetilde{\phi}_{n \ell}^{a}(r) .
$$

The projector functions must be orthonormalized as described by Blöchl. ${ }^{27}$ The potential $\bar{v}^{a}$ is chosen so that the local effective potential, $\widetilde{v}=\widetilde{v}^{\mathrm{H}}+\widetilde{v}_{\mathrm{xc}}+\bar{v}^{a}$, has the following shape for $r<r_{c}^{a}$ in the atomic reference state

$$
\widetilde{v}(r)=a^{a}+b^{a} r^{2}, \quad r<r_{c}^{a} .
$$

The constants $a^{a}$ and $b^{a}$ are found by requiring that $\bar{v}\left(r_{c}^{a}\right)$ $=0$ and $d \bar{v}(r) /\left.d r\right|_{r=r_{c}^{a}}=0$.

\section{APPENDIX B: COMPENSATION CHARGES}

For the compensation charges we use Gaussians

$$
\widetilde{g}_{\ell}^{a}(r)=\frac{1}{\sqrt{4 \pi}} \frac{\ell !}{(2 \ell+1) !}\left(4 \alpha^{a}\right)^{\ell+3 / 2} r^{\ell} e^{-\alpha^{a} r^{2}}
$$

and

$$
\hat{g}_{\ell}^{a}(r)=\frac{1}{\sqrt{4 \pi}} \frac{\ell !}{(2 \ell+1) !}\left(4 \hat{\alpha}^{a}\right)^{\ell+3 / 2} r^{\ell} e^{-\hat{\alpha}^{a} r^{2}} .
$$

With this choice for the compensation charges, the integral $V_{L L^{\prime}}^{a a^{\prime}}$ in Eq. (37) can be evaluated analytically. ${ }^{57}$ We choose the $\alpha$ 's so that $\alpha^{a}\left(r_{c}^{a}\right)^{2}=9.0$ and $\hat{\alpha}^{a}\left(\hat{r}_{c}^{a}\right)^{2}=22.0$.

\section{APPENDIX C: ATOMIC CONSTANTS}

By inserting Eqs. (7), (8), and (12) into Eqs. (19) and (20), we can reduce $E^{a}-\widetilde{E}^{a}$ to 


$$
\begin{aligned}
E^{a}-\widetilde{E}^{a}= & F^{a}+\sum_{i_{1} i_{2}} D_{i_{1} i_{2}}^{a} G_{i_{1} i_{2}}^{a}+\sum_{i_{1} i_{2} i_{3} i_{4}} D_{i_{1} i_{2} i_{i_{1} i_{2} i_{3} i_{4}}^{a}} D_{i_{3} i_{4}}^{a} \\
& +\sum_{L} Q_{L}^{a} J_{L}^{a}+\sum_{i_{1} i_{2}} \sum_{L} D_{i_{1} i_{2}}^{a} Q_{L}^{a} K_{i_{1} i_{2} L}^{a}+\sum_{L L^{\prime}} Q_{L}^{a} Q_{L^{\prime}}^{a} M_{L L^{\prime}}^{a} \\
& +\Delta E_{x c}^{a}\left(\left\{D_{i_{1} i_{2}}^{a}\right\}\right) .
\end{aligned}
$$

The integrals $F^{a}, G_{i_{1} i_{2}}^{a}, I_{i_{1} i_{2} i_{3} i_{4}}^{a}, J_{L}^{a}, K_{i_{1} i_{2} L}^{a}$, and $M_{L L^{\prime}}^{a}$ are given below and need only be computed once for each type of atom.

Inserting Eq. (14) into Eq. (C1) leads us to Eq. (21), where

$$
\begin{gathered}
A^{a}=F^{a}+\Delta^{a} K_{00}^{a}+\left(\Delta^{a}\right)^{2} N_{00,00}^{a} \\
B_{i_{1} i_{2}}^{a}=J_{i_{1} i_{2}}^{a}+\sum_{L} \Delta_{L i_{1} i_{2}}^{a} K_{L}^{a}+\Delta^{a} M_{i_{1} i_{2}, 00}^{a}+2 \sum_{L} \Delta_{L i_{1} i_{2}}^{a} N_{00,00}^{a} \Delta^{a} \\
C_{i_{1} i_{2} i_{3} i_{4}}^{a}=J_{i_{1} i_{2} i_{3} i_{4}}^{a}+\sum_{L} M_{i_{1} i_{2} L}^{a} \Delta_{L i_{3} i_{4}}^{a}+\sum_{L L^{\prime}} \Delta_{L i_{1} i_{2}}^{a} N_{L L^{\prime}}^{a} \Delta_{L^{\prime} i_{3} i_{4}}^{a} .
\end{gathered}
$$

We use the symmetrized $C_{i_{1} i_{2} i_{3} i_{4}}^{a}$,

$$
C_{i_{1} i_{2} i_{3} i_{4}}^{a} \leftarrow \frac{1}{2}\left(C_{i_{1} i_{2} i_{3} i_{4}}^{a}+C_{i_{3} i_{4} i_{1} i_{2}}^{a}\right) .
$$

The integrals for $F^{a}, G_{i_{1} i_{2}}^{a}, I_{i_{1} i_{2} i_{3} i_{4}}^{a}, J_{L}^{a}, K_{i_{1} i_{2} L}^{a}$, and $M_{L L^{\prime}}^{a}$ are (all integrals are limited to inside the augmentation spheres)

$$
\begin{aligned}
& F^{a}=\sum_{i}^{\text {core }} \int d \mathbf{r} \phi_{i}^{a, \text { core }}(\mathbf{r})\left(-\frac{1}{2} \nabla^{2}\right) \phi_{i}^{a, \text { core }}(\mathbf{r})-\int d \mathbf{r} \frac{n_{c}^{a}(r)}{r} \mathcal{Z}^{a} \\
& +\frac{1}{2} \int d \mathbf{r} v_{c}^{a}(r)\left[n_{c}^{a}(r)+\widetilde{n}_{c}^{a}(r)\right]-\int d \mathbf{r} \widetilde{n}_{c}^{a}(r) \bar{v}^{a}(r), \\
& I_{i_{1} i_{2}}^{a}=-\frac{1}{2} \int d \mathbf{r}\left[\phi_{i_{1}}^{a}(\mathbf{r}) \nabla^{2} \phi_{i_{2}}^{a}(\mathbf{r})-\widetilde{\phi}_{i_{1}}^{a}(\mathbf{r}) \nabla^{2} \widetilde{\phi}_{i_{2}}^{a}(\mathbf{r})\right] \\
& +\int d \mathbf{r}\left[v_{i_{1} i_{2}}^{a}(\mathbf{r}) n_{c}(r)+v_{c}^{a}(r) \widetilde{\phi}_{i_{1}}^{a}(\mathbf{r}) \widetilde{\phi}_{i_{2}}^{a}(\mathbf{r})\right] \\
& -\int d \mathbf{r} \frac{\phi_{i_{1}}^{a}(\mathbf{r}) \phi_{i_{2}}^{a}(\mathbf{r})}{r} \mathcal{Z}^{a}-\int d \mathbf{r} \widetilde{\phi}_{i_{1}}^{a}(\mathbf{r}) \widetilde{\phi}_{i_{2}}^{a}(\mathbf{r}) \bar{v}^{a}(r),
\end{aligned}
$$

$$
J_{i_{1} i_{2} i_{3} i_{4}}^{a}=\frac{1}{2} \int d \mathbf{r} v_{i_{1} i_{2}}^{a}(\mathbf{r})\left[\phi_{i_{3}}^{a}(\mathbf{r}) \phi_{i_{4}}^{a}(\mathbf{r})+\widetilde{\phi}_{i_{3}}^{a}(\mathbf{r}) \widetilde{\phi}_{i_{4}}^{a}(\mathbf{r})\right],
$$

$$
K_{L}^{a}=-\int d \mathbf{r} \int d \mathbf{r}^{\prime} \frac{\widetilde{g}_{L}^{a}\left(\mathbf{r}^{\prime}\right)}{\left|\mathbf{r}-\mathbf{r}^{\prime}\right|} \widetilde{n}_{c}^{a}(r),
$$

$$
M_{i_{1} i_{2} L}^{a}=-\int d \mathbf{r} \int d \mathbf{r}^{\prime} \frac{\widetilde{g}_{L}^{a}\left(\mathbf{r}^{\prime}\right)}{\left|\mathbf{r}-\mathbf{r}^{\prime}\right|} \widetilde{\phi}_{i_{1}}^{a}(\mathbf{r}) \widetilde{\phi}_{i_{2}}^{a}(\mathbf{r})
$$

and

$$
N_{L L^{\prime}}^{a}=-\frac{1}{2} \int d \mathbf{r} \int d \mathbf{r}^{\prime} \frac{\widetilde{g}_{L}^{a}\left(\mathbf{r}^{\prime}\right)}{\left|\mathbf{r}-\mathbf{r}^{\prime}\right|} \widetilde{g}_{L^{\prime}}^{a}(\mathbf{r}) .
$$

The potentials $v_{i_{1} i_{2}}^{a}(\mathbf{r})$ and $v_{c}^{a}(r)$ are defined as

$$
v_{i_{1} i_{2}}^{a}(\mathbf{r})=\int d \mathbf{r}^{\prime} \frac{\phi_{i_{1}}^{a}\left(\mathbf{r}^{\prime}\right) \phi_{i_{2}}^{a}\left(\mathbf{r}^{\prime}\right)-\widetilde{\phi}_{i_{1}}^{a}\left(\mathbf{r}^{\prime}\right) \widetilde{\phi}_{i_{2}}^{a}\left(\mathbf{r}^{\prime}\right)}{\left|\mathbf{r}-\mathbf{r}^{\prime}\right|}
$$

and

$$
v_{c}^{a}(r)=\int d \mathbf{r}^{\prime} \frac{n_{c}^{a}\left(r^{\prime}\right)-\widetilde{n}_{c}^{a}\left(r^{\prime}\right)}{\left|\mathbf{r}-\mathbf{r}^{\prime}\right|} .
$$

It is advantageous to decompose $v_{i_{1} i_{2}}^{a}(\mathbf{r})$ into angular momentum contributions as

$$
v_{i_{1} i_{2}}^{a}(\mathbf{r})=\sum_{L} v_{i_{1} i_{2} \ell}^{a}(r) Y_{L}(\mathbf{r})
$$

and solve

$$
\begin{aligned}
\nabla^{2}\left[v_{i_{1} i_{2} \ell}^{a}(r) Y_{L}(\hat{\mathbf{r}})\right]= & -4 \pi G_{L_{1} L_{2}}^{L} Y_{L}(\hat{\mathbf{r}}) \times\left[\phi_{n_{1} \ell_{1}}^{a}(r) \phi_{n_{2} \ell_{2}}^{a}(r)\right. \\
& \left.-\widetilde{\phi}_{n_{1} \ell_{1}}^{a}(r) \widetilde{\phi}_{n_{2} \ell_{2}}^{a}(r)\right],
\end{aligned}
$$

where

$$
G_{L_{1} L_{2}}^{L}=\int_{0}^{\pi} \sin \theta d \theta \int_{0}^{2 \pi} d \phi Y_{L}(\theta, \phi) Y_{L_{1}}(\theta, \phi) Y_{L_{2}}(\theta, \phi)
$$

is a Gaunt coefficient.

\section{APPENDIX D: HAMILTONIAN}

The discretized Hamiltonian [Eq. (43)] depends on $\widetilde{v}_{G}$ and $H_{i_{1} i_{2}}^{a}$. The local effective potential on the coarse grid, $\widetilde{v}_{G}$ $=\left(V_{f} / V_{c}\right) \Sigma_{g} I_{g G} \widetilde{v}_{g}$, is a restriction of the local effective potential on the fine grid

$$
\widetilde{v}_{g}=\widetilde{v}_{g}^{\mathrm{H}}+\sum_{a} \hat{v}_{g}^{a}+\sum_{a} \bar{v}_{g}^{a}+\frac{1}{V_{f}} \frac{\partial E_{\mathrm{xc}}}{\partial \widetilde{n}_{g}},
$$

and the atomic Hamiltonian $H_{i_{1} i_{2}}^{a}$ is

$$
H_{i_{1} i_{2}}^{a}=\sum_{L} \Delta_{L i_{1} i_{2}}^{a} W_{L}^{a}+\frac{\partial \Delta E_{x c}^{a}}{\partial D_{i_{1} i_{2}}^{a}}+B_{i_{1} i_{2}}^{a}+2 \sum_{i_{3} i_{4}} C_{i_{1} i_{2} i_{3} i_{4}}^{a} D_{i_{3} i_{4}}^{a},
$$

where

$$
W_{L}^{a}=\frac{\partial \widetilde{E}}{\partial Q_{L}^{a}}=V_{f} \sum_{g} \widetilde{v}_{g}^{\mathrm{H}} \hat{g}_{L g}^{a}+V_{f} \sum_{g} \tilde{n}_{g} \hat{v}_{L g}^{a}+\sum_{a^{\prime}} \sum_{L^{\prime}} V_{L L^{\prime}}^{a a^{\prime}} Q_{L^{\prime}}^{a^{\prime}} .
$$


${ }^{1}$ P. Hohenberg and W. Kohn, Phys. Rev. 136, B864 (1964).

${ }^{2}$ W. Kohn and L. J. Sham, Phys. Rev. 140, A1133 (1965).

${ }^{3}$ J. R. Chelikowsky, N. Troullier, K. Wu, and Y. Saad, Phys. Rev. B 50, 11355 (1994).

${ }^{4}$ A. P. Seitsonen, M. J. Puska, and R. M. Nieminen, Phys. Rev. B 51, 14057 (1995).

${ }^{5}$ T. Hoshi, M. Arai, and T. Fujiwara, Phys. Rev. B 52, R5459 (1995).

${ }^{6}$ E. L. Briggs, D. J. Sullivan, and J. Bernholc, Phys. Rev. B 54, 14362 (1996).

${ }^{7}$ J. Bernholc, E. L. Briggs, D. J. Sullivan, C. J. Brabec, M. B. Nardelli, K. Rapcewicz, C. Roland, and M. Wensell, Int. J. Quantum Chem. 65, 531 (1997).

${ }^{8}$ F. Ancilotto, P. Blandin, and F. Toigo, Phys. Rev. B 59, 7868 (1999).

${ }^{9}$ J. Bernholc, E. L. Briggs, C. Bungaro, M. B. Nardelli, J.-L. Fattebert, K. Rapcewicz, C. Roland, W. G. Schmidt, and Q. Zhao, Phys. Status Solidi A 217, 685 (2000).

${ }^{10}$ T. L. Beck, Rev. Mod. Phys. 72, 1041 (2000).

${ }^{11}$ J. Wang and T. L. Beck, J. Chem. Phys. 112, 9223 (2000).

${ }^{12}$ M. Heiskanen, T. Torsti, M. J. Puska, and R. M. Nieminen, Phys. Rev. B 63, 245106 (2001).

${ }^{13}$ U. V. Waghmare, H. Kim, I. J. Park, N. Modine, P. Maragakis, and E. Kaxiras, Comput. Phys. Commun. 137, 341 (2001).

${ }^{14}$ H. Takahashi, T. Hori, T. Wakabayashi, and T. Nitta, J. Phys. Chem. 105, 4351 (2001).

${ }^{15}$ M. A. L. Marques, A. Castro, G. F. Bertsch, and A. Rubio, Comput. Phys. Commun. 151, 60 (2003).

${ }^{16}$ R. Schmid, J. Comput. Chem. 25, 799 (2004).

${ }^{17}$ F. Shimojo, R. K. Kalia, A. Nakano, and P. Vashishta, Comput. Phys. Commun. 140, 303 (2001).

${ }^{18}$ Y. Liu, D. A. Yarne, and M. E. Tuckerman, Phys. Rev. B 68, 125110 (2003).

${ }^{19}$ A. Brandt, Math. Comput. 31, 333 (1977).

${ }^{20}$ A. A. Mostofi, C.-K. Skylaris, P. D. Haynes, and M. C. Payne, Comput. Phys. Commun. 147, 788 (2002).

${ }^{21}$ G. Galli and M. Parrinello, Phys. Rev. Lett. 69, 3547 (1992).

${ }^{22}$ S. Goedecker, M. Boulet, and T. Deutsch, Comput. Phys. Commun. 154, 105 (2003).

${ }^{23}$ P. E. Blöchl, J. Chem. Phys. 103, 7422 (1995).

${ }^{24}$ G. J. Martyna and M. E. Tuckerman, J. Chem. Phys. 110, 2810 (1999).

${ }^{25}$ D. Vanderbilt, Phys. Rev. B 41, R7892 (1990).

${ }^{26}$ K. Laasonen, A. Pasquarello, R. Car, C. Lee, and D. Vanderbilt, Phys. Rev. B 47, 10142 (1992).

${ }^{27}$ P. E. Blöchl, Phys. Rev. B 50, 17953 (1994).

${ }^{28}$ P. E. Blöchl, C. J. Forst, and J. Schimpl, Bull. Mater. Sci. 26, 33 (2003).
${ }^{29}$ N. A. W. Holzwarth, G. E. Matthews, R. B. Dunning, A. R. Tackett, and Y. Zeng, Phys. Rev. B 55, 2005 (1997).

${ }^{30}$ G. Kresse and D. Joubert, Phys. Rev. B 59, 1758 (1999).

${ }^{31}$ M. Valiev and J. H. Weare, J. Phys. Chem. 103, 10588 (1999).

${ }^{32}$ A. R. Tackett, N. A. W. Holzwarth, and G. E. Matthews, Comput. Phys. Commun. 135, 348 (2001).

${ }^{33}$ G. Kresse and J. Furthmuller, Phys. Rev. B 54, 11169 (1996).

${ }^{34}$ P. Pulay, Chem. Phys. Lett. 73, 393 (1980).

${ }^{35}$ D. M. Wood and A. Zunger, J. Phys. A 18, 1343 (1985).

${ }^{36}$ J. Fliege and U. Maier, IMA J. Numer. Anal. 19, 317 (1999); http://www.mathematik.uni-dortmund.de/lsx/research/projects/ fliege/nodes/nodes.html.

${ }^{37}$ Although we find this method to be the simplest for GGA functionals, this is probably the most complicated part of the code.

${ }^{38}$ Y. C. Zheng and J. E. Almlöf, J. Mol. Struct. 388, 277 (1996).

${ }^{39}$ C. Berghold, J. Hutter, and M. Parrinello, Theor. Chem. Acc. 99, 344 (1998).

${ }^{40}$ T. Ono and K. Hirose, Phys. Rev. Lett. 82, 5016 (1999).

${ }^{41}$ M. C. Payne, M. P. Teter, D. C. Allan, T. A. Arias, and J. D. Joannopoulos, Rev. Mod. Phys. 64, 1045 (1992).

${ }^{42}$ T. Korhonen, M. J. Puska, and R. M. Nieminen, Phys. Rev. B 54, 15016 (1996).

${ }^{43}$ J. A. White and D. M. Bird, Phys. Rev. B 50, 4954 (R) (1994).

${ }^{44}$ J. P. Perdew, K. Burke, and M. Ernzerhof, Phys. Rev. Lett. 77, 3865 (1996).

${ }^{45}$ F. W. Kutzler and G. S. Painter, Phys. Rev. Lett. 59, 1285 (1987).

${ }^{46}$ S. Kurth, J. P. Perdew, and P. Blaha, Int. J. Quantum Chem. 75, 889 (1999).

${ }^{47}$ Y. Zhang and W. Yang, Phys. Rev. Lett. 80, 890 (1998).

${ }^{48}$ I. P. Daykov, T. A. Arias, and T. D. Engeness, Phys. Rev. Lett. 90, 216402 (2003).

${ }^{49}$ J. P. Perdew and Y. Wang, Phys. Rev. B 45, 13244 (1992).

${ }^{50}$ S. R. Bahn and K. W. Jacobsen, Comput. Sci. Eng. 4, 56 (2002).

${ }^{51}$ B. Hammer, L. B. Hansen, and J. K. Nørskov, Phys. Rev. B 59, 7413 (1999).

${ }^{52}$ The Dacapo code is freely available at http://www.fysik.dtu.dk/ campos.

${ }^{53}$ There are 2897 complex plane-wave coefficients, but only half of the coefficients need to be stored, because the wave function can be chosen as real.

${ }^{54}$ N. Troullier, J. R. Chelikowsky, and Y. Saad, Solid State Commun. 93, 225 (1995).

${ }^{55}$ The code is written in the Python and $\mathrm{C}++$ programming languages, and is freely available at http://www.fysik.dtu.dk/ campos/gridpaw.

${ }^{56}$ G. P. Kerker, Phys. Rev. B 23, 3082 (1981).

${ }^{57}$ S. Obara and A. Saika, J. Chem. Phys. 84, 3963 (1986). 